\title{
A systematic review of vasopressor blood pressure targets in critically ill adults with hypotension
}

\section{Une revue systématique des cibles de tension artérielle sous vasopresseurs chez des adultes gravement malades atteints d'hypotension}

\author{
Mathieu Hylands, MD, MSc • Morten Hylander Moller, MD • Pierre Asfar, MD, PhD • \\ Augustin Toma, BSc • Anne Julie Frenette, BPharm, MSc $\cdot$ Nicolas Beaudoin, MD, MSc • \\ Émilie Belley-Côté, MD, MSc · Frédérick D’Aragon, MD, MSc · Jon Henrik Laake, MD, PhD • \\ Reed Alexander Siemieniuk, MD • Emmanuel Charbonney, MD, PhD • \\ François Lauzier, MD, MSc • Joey Kwong, PhD • Bram Rochwerg, MD, MSc • \\ Per Olav Vandvik, MD, PhD • Gordon Guyatt, MD, MSc • François Lamontagne, MD, MSc $\mathbb{D}$ \\ Received: 4 November 2016/Revised: 13 March 2017/Accepted: 11 April 2017/Published online: 11 May 2017 \\ (C) Canadian Anesthesiologists' Society 2017
}

\begin{abstract}
Purpose Clinicians must balance the risks from hypotension with the potential adverse effects of vasopressors. Experts have recommended a mean arterial pressure (MAP) target of at least $65 \mathrm{mmHg}$, and higher in older patients and in patients with chronic hypertension or atherosclerosis. We conducted a systematic review of randomized-controlled trials comparing higher vs lower blood pressure targets for vasopressor therapy administered to hypotensive critically ill patients.

Methods We searched MEDLINE®, EMBASE ${ }^{T M}$, and the Cochrane Central Register of Controlled Trials for studies of higher vs lower blood pressure targets for vasopressor
\end{abstract}

This article is accompanied by an editorial. Please see Can J Anesth 2017; 64: this issue.

M. Hylands, MD, MSc

Department of Surgery, Université de Sherbrooke, Sherbrooke, QC, Canada

M. H. Moller, MD

Copenhagen University Hospital Rigshospitalet, Copenhagen, Denmark

P. Asfar, MD, PhD

Centre Hospitalier Universitaire d'Angers, Angers, France

A. Toma, BSc · É. Belley-Côté, MD, MSc .

R. A. Siemieniuk, MD - B. Rochwerg, MD, MSc .

G. Guyatt, MD, MSc

Department of Clinical Epidemiology and Biostatistics,

McMaster University, Hamilton, ON, Canada therapy in critically ill hypotensive adult patients. Two reviewers independently assessed trial eligibility based on titles and abstracts, and they then selected full-text reports. Outcomes, subgroups, and analyses were prespecified. We used GRADE (Grading of Recommendations Assessment, Development and Evaluation) to rate the overall confidence in the estimates of intervention effects.

Results Of 8001 citations, we retrieved 57 full-text articles and ultimately included two randomized-controlled trials (894 patients). Higher blood pressure targets were not associated with lower mortality (relative risk [RR], 1.05; 95\% confidence interval [CI], 0.90 to $1.23 ; P=0.54)$, and neither age $(P=0.17)$ nor chronic hypertension $(P=0.32)$ modified the overall effect. Nevertheless, higher blood pressure targets were associated with a greater risk of

A. J. Frenette, BPharm, MSc - E. Charbonney, MD, PhD Centre de recherche de l'Hôpital du Sacré-Coeur de Montréal, Université de Montréal, Montréal, QC, Canada

N. Beaudoin, MD, MSc · F. D'Aragon, MD, MSc

Department of Anesthesiology, Université de Sherbrooke, Sherbrooke, QC, Canada

É. Belley-Côté, MD, MSc · F. Lamontagne, MD, MSc Department of Medicine, Université de Sherbrooke, Sherbrooke, Canada

F. D'Aragon, MD, MSc · F. Lamontagne, MD, MSc ( $\square)$ Centre de recherche du CHU de Sherbrooke, Université de Sherbrooke, Sherbrooke, QC, Canada e-mail: francois.lamontagne@usherbrooke.ca 
new-onset supraventricular cardiac arrhythmia $(R R, 2.08$; 95\% CI, 1.28 to 3.38; $P<0.01$ ).

Conclusion Current evidence does not support a MAP target $>70 \mathrm{mmHg}$ in hypotensive critically ill adult patients requiring vasopressor therapy.

\section{Résumé}

Objectif Les cliniciens doivent équilibrer les risques liés à l'hypotension aux complications potentielles des vasopresseurs. Des experts ont recommandé de cibler une tension artérielle moyenne (TAM) d'au moins 65 $\mathrm{mmHg}$, et une TAM plus élevée chez les patients atteints d'hypertension chronique, d'athérosclérose ou plus âgés. Nous avons réalisé une revue systématique des études randomisées contrôlées comparant des cibles de tension artérielle plus élevées à plus basses chez des patients hypotendus en état critique recevant un traitement vasopresseur.

Méthode Nous avons fait des recherches dans les bases de données Medline, EMBASE et dans le registre central des études contrôlées Cochrane afin d'en extraire les études comparant des cibles de tension artérielle plus élevées ou plus basses chez des patients adultes hypotendus et en état critique recevant un traitement vasopresseur. Deux examinateurs ont évalué de façon indépendante l'éligibilité des études selon leur titre et leur résumé, puis sélectionné les articles intégraux. Les critères d'évaluation, sous-groupes et analyses étaient spécifiés au préalable. Nous avons utilisé le système GRADE (Grading of Recommendations Assessment, Development and Evaluation) afin d'évaluer la confiance globale dans les estimations des effets de l'intervention.

Résultats Parmi les 8001 citations, nous avons extrait 57 articles intégraux et finalement inclus deux études randomisées contrôlées (894 patients). Les cibles de

\section{J. H. Laake, MD, PhD}

Oslo Universitetssykehus Ulleval, Oslo, Norway

F. Lauzier, MD, MSc

Centre de Recherche du CHU de Québec - Université Laval, Population Health and Optimal Health Practices Research Unit (Trauma - Emergency - Critical Care Medicine), Quebec, QC, Canada

J. Kwong, $\mathrm{PhD}$

Wuhan University, Center for Evidence-Based and Translational Medicine Zhongnan Hospital, Wuhan, China

B. Rochwerg, MD, MSc

Department of Medicine, Faculty of Health Sciences, McMaster University, Hamilton, ON, Canada

P. O. Vandvik, MD, PhD

Norwegian Knowledge Centre for the Health Services, Oslo, Norway tension artérielle plus élevées n'étaient pas associées à une mortalité plus basse (risque relatif [RR] 1,05; intervalle de confiance [IC] $95 \%, 0,90$ à 1,23; $P=$ $0,54)$, et ni l'âge $(P=0,17)$ ni l'hypertension chronique $(P=0,32) n$ 'ont modifié l'effet global. Cependant, les cibles de tension artérielle plus élevées étaient associées à un risque plus élevé de nouvelle apparition d'une arythmie cardiaque supraventriculaire (RR, 2,08; IC $95 \%, 1,28$ à 3,38; $P<0,01)$.

Conclusion Les données probantes actuelles n'appuient pas une cible de TAM supérieure à $70 \mathrm{mmHg}$ chez les patients adultes hypotendus et gravement malades nécessitant un traitement vasopresseur.

The global burden of critical illness exceeds the cumulative mortality of breast cancer, HIV, and asthma combined. ${ }^{1}$ In the critically ill, hypotension is common and often lethal when extreme, progressive, or persistent. Vasopressors are used to compensate for excessive vasodilation or, when facing imminent circulatory arrest regardless of the cause of hypotension, to raise blood pressure for a short period while other corrective measures are implemented. ${ }^{2}$ Nevertheless, vasopressor-induced vasoconstriction can compromise blood perfusion at a microvascular level, even when blood pressure is kept within normal ranges. Vasopressors also have numerous pleiotropic effects that are not easy to predict or measure, and some of these can be harmful. ${ }^{3}$

Notwithstanding, clinicians must balance the risks from hypotension with the potential adverse effects of vasopressors. Experts have recommended a mean arterial pressure (MAP) target of at least $65 \mathrm{mmHg}$, and higher in older patients and in patients with chronic hypertension or atherosclerosis. ${ }^{4}$ Recent observational evidence suggests that patients exposed to higher vasopressor doses have a higher risk of adverse cardiovascular events. ${ }^{5}$

In 2016, the Canadian Critical Care Society and the Scandinavian Society of Anaesthesiology and Intensive Care undertook joint guidelines on targets for vasopressor use. To inform these guidelines, we updated a systematic review published in $2015 .{ }^{6}$ Following recommendations for trustworthy guidelines, ${ }^{7}$ we submitted the protocol of this systematic review to the guideline panel whose recommendations bore on the final version of the protocol. Accordingly, the research question was broadened beyond the previous systematic review to include all forms of hypotension. This work aims to answer the following question: In adult critically ill patients with hypotension requiring vasopressor support, should we prescribe higher (MAP 75-85 $\mathrm{mmHg}$ ) vs lower (MAP 60-70 $\mathrm{mmHg}$ ) blood pressure targets? Our 
hypothesis was that higher MAP targets would not be associated with clinically measurable benefits.

\section{Methods}

The guidelines were created according to standards for trustworthy guidelines in collaboration with the MAGIC WikiRecs project. ${ }^{8}$ After consultation with the members of the guideline panel, we registered this systematic review on PROSPERO (CRD42016033438) and present the results according to PRISMA guidance. ${ }^{9}$

\section{Search strategy and study selection}

We searched MEDLINE®) (from 1946 to October 12, 2016), EMBASE ${ }^{\mathrm{TM}}$ (from 1980 to October 12, 2016), and the Cochrane Central Register of Controlled Trials for randomized-controlled trials of higher vs lower blood pressure targets for vasopressor therapy in adult patients who are in shock or hypotensive (as defined by the investigators). The studies were required to provide information on at least one clinically important outcome (defined as mortality, quality of life after hospital discharge, use of renal replacement therapy, duration of intensive care unit (ICU) or hospital stay, or vasopressorinduced adverse events). We excluded crossover designs, studies conducted in pediatric populations, trials of vasopressors that are not routinely used, and studies in which the duration of the experiment was designed for less than $<24$ hr by design (i.e., studies could be included where patients were treated for less than $<24 \mathrm{hr}$ in included studies, but studies were excluded where patients were followed for brief periods - were excluded because short protocols were not expected to influence clinically relevant outcomes). There was no exclusion based on the language of the published report or the quality of evidence. A detailed search strategy appears in the Appendix.

We also manually searched published editorials, reviews, and the reference lists of identified articles, as well as proceedings from the annual meetings (20052016) of the American Thoracic Society, the Society of Critical Care Medicine, the European Society of Intensive Care Medicine, the International Symposium on Intensive Care and Emergency Medicine, the American Association for the Surgery of Trauma, the Eastern Association for the Surgery of Trauma, the Shock Society, the European Shock Society and the American College of Chest Physicians.

Two reviewers independently assessed trial eligibility based on titles and abstracts, and they then selected fulltext reports. We contacted authors for further information when uncertainty persisted about eligibility.
Outcomes

All outcomes included in this review were prespecified. The primary outcome was all-cause short-term mortality, defined as the longest reported follow-up within 90 days, including ICU and hospital mortality. Secondary outcomes included long-term mortality, defined by a more distant time point (e.g., six months), early acute renal replacement therapy (within 90 days), late chronic renal replacement therapy (after 90 days), duration of renal replacement therapy (days), duration of mechanical ventilation (days), fluid use (cumulative volume received), blood product requirements (cumulative volume received), acute kidney injury, newonset cardiac arrhythmia, digit or limb or skin ischemia, mesenteric ischemia, myocardial ischemia, quality of life, and neurological outcome at longest reported follow-up.

Data abstraction

We prepared and piloted data extraction forms before launching the study. Reviewers followed written instructions that were developed a priori in order to standardize data extraction. In teams of two, reviewers then independently collected information on study design, patient population, interventions, and outcomes.

\section{Statistical analysis}

All analyses were conducted according to the predefined statistical analysis plan outlined in the protocol (CRD42016033438).

For all outcomes, we compared patients treated with a higher blood pressure target $v s$ patients treated with a lower blood pressure target. Using the Review Manager 5.3 software (Cochrane Collaboration), we meta-analyzed the included studies using the random-effects model described by DerSimonian and Laird, and individual study weights were measured using the inverse variance method. ${ }^{10}$ For dichotomous data, we present pooled summary effect measures as risk ratios (RRs), with $95 \%$ confidence intervals (CIs) ${ }^{11}$ for relative effects and number needed to treat, and risk difference by assuming the baseline risk for each outcome without the intervention is the risk in the control group for absolute effects. ${ }^{12}$ When appropriate, we applied a continuity correction of zero outcome scenarios. Effect sizes for continuous variables are presented as mean differences (MDs) with 95\% CIs.

We assessed study heterogeneity qualitatively by considering whether or not study populations, interventions, and settings were comparable across reports. We performed a Chi square test for homogeneity and assessed heterogeneity quantitatively using Higgins and Thompson's $I^{2}$ statistic. 
Subgroup analyses

We used random effects models to perform two subgroup analyses of the primary outcome and report the statistical interaction between study-level subgroup-defining variables and the intervention (high $v s$ low blood pressure target). The subgroup-defining variables were 1) presence or absence of baseline hypertension - hypothesizing that hypertensive patients will benefit more from high blood pressure targets, and 2) age $\geq 65 \mathrm{yr} v s<65 \mathrm{yr}-$ hypothesizing that more elderly patients benefit from lower blood pressure targets. Both the subgroup analyses and our hypothesized direction of effect were prespecified. Study-level data on other prespecified subgroups (congestive heart failure, etiology of hypotension, illness severity, and risk of bias) were not available.

Risk of bias assessment for individual studies

We assessed risk of bias in randomized-controlled trials at the study level independently for each outcome using a modified version (includes a systematic assessment for risks of bias associated with stopping a trial before the planned sample size is reached) ${ }^{13}$ of the Cochrane Collaboration's instrument. ${ }^{12,14}$ The instrument addresses the following domains: allocation sequence concealment, blinding of participants and caregivers, blinding of data collectors, blinding for outcome assessment, blinding of data analysts, loss to follow-up, selective outcome reporting, and other risks of bias. Studies with one or more domains assessed as a potential source of bias were considered overall at high risk of bias. We assessed the overall quality of the data for each individual outcome using the Grading of Recommendations Assessment, Development and Evaluation (GRADE) approach. ${ }^{15}$

Publication bias and confidence in the evidence

A statistical assessment of the risk of publication bias was planned if there were at least ten included studies. ${ }^{16} \mathrm{We}$ used GRADE to rate the overall confidence in the estimates of intervention effects. ${ }^{15}$ In this method, quality of evidence is rated "very low," "low," "moderate" or "high". Randomized-controlled trials begin as high-quality evidence, while observational studies begin as low-quality evidence. Quality of evidence can be rated down for risk of bias, ${ }^{17}$ imprecision, ${ }^{18}$ inconsistency, ${ }^{19}$ indirectness, ${ }^{20}$ and likelihood of publication bias. ${ }^{21}$ Observational studies can be rated up in the presence of a large magnitude of the association, a dose-response gradient, or if all unaccounted confounders increase confidence in estimates of effect. We assessed the risk of random errors (imprecision) by conducting trial sequential analyses (TSA). ${ }^{22}$ Trial sequential analyses is a sample size calculation (interim analysis) for cumulative meta-analyses that widens the confidence intervals when data are too sparse to draw firm conclusions. For each TSA, we report the required sample size from all trials and the proportion of this sample size already accrued.

\section{Results}

We retrieved 57 full-text articles from 8001 citations and ultimately included two randomized controlled trials. ${ }^{23,24}$ We identified one ongoing trial (NCT01473498). A PRISMA flowchart illustrates the selection process (Fig. 1).

Study characteristics

Table 1 illustrates study characteristics. The included trials recruited 894 patients from France, Canada, and the United States. In both trials, norepinephrine was the most common vasopressor, and the higher MAP targets were $15 \mathrm{mmHg}$ above the lower MAP targets. Adherence to protocol was superior in the higher MAP arms in both trials (i.e., actual MAP values were above the upper limit of the prescribed range in the lower MAP arms only). While one trial did not restrict patient eligibility to septic shock, this was the most common admission diagnosis in both trials. Differences between trials included the duration of vasopressor therapy allowed before randomization (longer in the study by Lamontagne et al.) and the specification of a minimal vasopressor dose at baseline (required in the study by Asfar et al. only). Excluded clinical experiments are summarized in the Appendix.

\section{Risk of bias}

In both trials, allocation was concealed; analyses respected the intention-to-treat principle; follow-up for the primary endpoint was complete; and enrolment was not stopped early because of evidence of benefit or harm. Nevertheless, in both trials, caregivers (including bedside clinicians) were aware of the allocated study arm. Assessors for arrhythmia occurring during the five-day intervention were blinded in the study by Asfar et al. We considered overall risk of bias to be high in both trials due to lack of blinding.

\section{Mortality}

Fig. 2 illustrates forest plots for the primary outcome. Higher blood pressure targets were not associated with lower short-term mortality (RR, 1.05 ; $95 \%$ CI, 0.90 to 1.23 ; $P=0.54$; low confidence) or long-term mortality (RR, $1.13 ; 95 \% \mathrm{CI}, 0.72$ to $1.77 ; P=0.60$; low confidence). Neither age (interaction test, $P=0.17$ ) nor chronic 


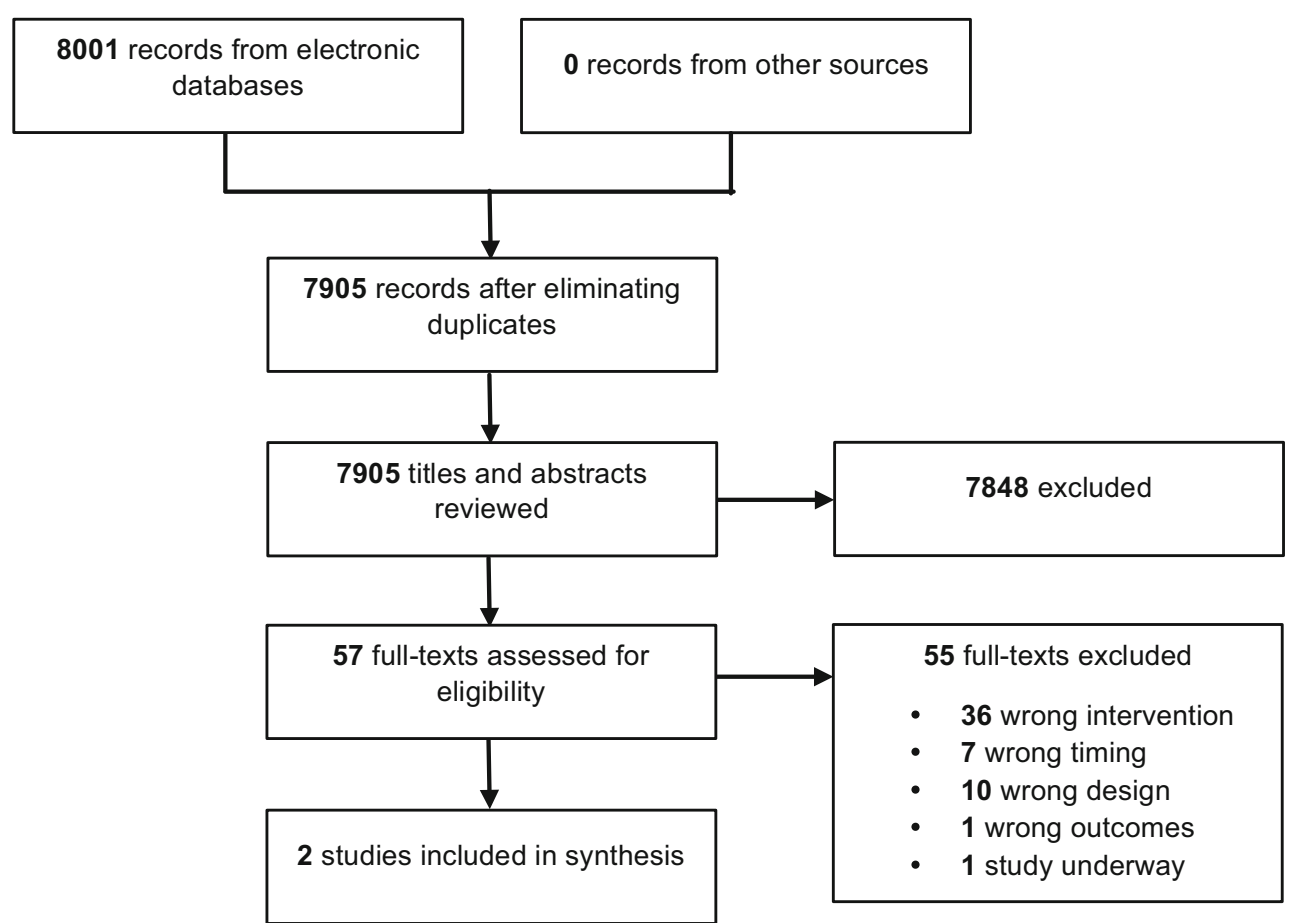

Fig. 1 PRISMA flowchart

hypertension (interaction test, $P=0.32$ ) modified the effect of higher blood pressure targets on mortality. Trial sequential analyses estimates were consistent with the primary analysis, but they highlighted that $82 \%$ of the required information size has been accrued.

Secondary outcomes

Higher blood pressure targets did not result in important differences in fluid balance over the first five ICU days (MD, $1.15 \mathrm{~L} ; 95 \% \mathrm{CI},-2.03$ to $4.33 ; P=0.48$; low confidence), use of acute renal replacement therapy (RR, $0.96 ; 95 \% \mathrm{CI}, 0.80$ to $1.14 ; P=0.61$; low confidence), duration of renal replacement therapy (MD, -0.41 days; $95 \% \mathrm{CI},-1.27$ to $0.45 ; P=0.35$; low confidence), or number of ventilator-free days (MD, -0.84 days; $95 \% \mathrm{CI}$, -2.28 to $0.60 ; P=0.25$; low confidence). There were no apparent differences in the risk of digit or limb or skin ischemia, mesenteric ischemia, myocardial ischemia, or ventricular arrhythmia. Nevertheless, higher blood pressure targets were associated with a greater risk of new-onset supraventricular cardiac arrhythmia (RR, 2.08; 95\% CI, 1.28 to $3.38 ; P<0.01$; low confidence) (Table 2).

The long-term neurological outcome, reported as the median [interquartile range] change from baseline to six months in the alpha Functional Independence Measure score among survivors, was reported in only one study $(n=$
118) and was identical in both groups $(0[-10-0])$. Neither study reported quality-of-life measures.

Quality of the evidence and publication bias

A summary of the evidence is presented in Table 2. Confidence in the effect estimates was low for all outcomes due to risk of bias and imprecision. We could not conduct statistical analyses to quantify the risk of publication bias.

\section{Discussion}

The results of this systematic review, which includes data from a trial published in 2016, do not suggest that higher blood pressure targets in adult critically ill patients needing vasopressors modify mortality overall. Further, current evidence does not support increasing MAP targets for patients who have chronic hypertension, as suggested by the 2013 Surviving Sepsis Campaign Guidelines. ${ }^{4}$ In contrast, we found that higher blood pressure targets might acutely increase the risk for supraventricular arrhythmia, which echoes the results of recent observational studies. ${ }^{5}$ These results underscore the absence of supportive evidence for individualized therapy based on the presence of chronic hypertension or patient age. ${ }^{4}$ Using this meta-analysis and considering all the 
Table 1 Included studies

\begin{tabular}{|c|c|c|}
\hline Study, year (ref) & Asfar, 2014(23) & Lamontagne, 2016(24) \\
\hline Location, number of sites & France, 29 & Canada, 10; United States, 1 \\
\hline Patients, $n$ & 776 & 118 \\
\hline Age, yr mean (SD) & $65(14)$ & $65(13)$ \\
\hline Severity score used, mean (SD) & SAPS II, 57 (16) & APACHE II, 25 (7) \\
\hline Inclusion criteria & $\begin{array}{l}>18 \text { years of age, septic shock refractory to fluid } \\
\quad \text { resuscitation, baseline norepinephrine dose } \geq 0.1 \\
\mu \mathrm{g} \cdot \mathrm{kg}^{-1} \cdot \mathrm{min}^{-1},<6 \mathrm{hr} \text { of vasopressor initiation }\end{array}$ & $\begin{array}{l}>16 \text { years of age, receiving vasopressors for } \\
\text { presumed vasodilatory shock despite fluid } \\
\text { resuscitation, vasopressor therapy for at least } 6 \mathrm{hr}\end{array}$ \\
\hline Exclusion criteria & $\begin{array}{l}\text { Legal protection, no affiliation with the French } \\
\text { healthcare system, pregnancy, participation in a } \\
\text { competing trial, do not resuscitate order }\end{array}$ & $\begin{array}{l}\text { Vasopressor therapy ongoing }>24 \mathrm{hr} \text {, expected to die } \\
<48 \mathrm{hr} \text {, indication for vasopressors is cardiogenic, } \\
\text { hemorrhagic, neurogenic shock, post-cardiac } \\
\text { surgery, or unrelated to hypotension }{ }^{1}\end{array}$ \\
\hline Admission diagnosis sepsis, $n(\%)$ & $776(100)$ & $83(70)^{2}$ \\
\hline \multicolumn{3}{|l|}{ Source of infection, $n(\%)$} \\
\hline Lung & $402(52)$ & $83(18)$ \\
\hline Abdomen & $132(17)$ & $11(9)$ \\
\hline Urinary tract & $88(11)$ & $8(7)$ \\
\hline Other & $145(19)$ & $43(36)$ \\
\hline \multirow[t]{2}{*}{ Intervention } & Target MAP $80-85 \mathrm{mmHg}$ & Target MAP 75-80 mmHg \\
\hline & $\begin{array}{l}\text { First-line vasopressor: epinephrine ( } 1 \text { centre) or } \\
\text { norepinephrine ( } 28 \text { centres) } \\
\text { Duration: maximum } 5 \text { days or until patient weaned } \\
\quad \text { from vasopressors } \\
n=388\end{array}$ & $\begin{array}{l}\text { Vasopressor: norepinephrine, vasopressin, } \\
\text { phenylephrine, epinephrine, and/or dopamine } \\
\text { Duration: up to } 28 \text { days } \\
n=58\end{array}$ \\
\hline \multirow[t]{3}{*}{ Control } & Target MAP 65-70 mmHg & Target MAP 60-65 mmHg \\
\hline & $\begin{array}{l}\text { First-line vasopressor: epinephrine ( } 1 \text { centre) or } \\
\text { norepinephrine ( } 28 \text { centres) }\end{array}$ & $\begin{array}{l}\text { Vasopressor: norepinephrine, vasopressin, } \\
\text { phenylephrine, epinephrine, and/or dopamine }\end{array}$ \\
\hline & $\begin{array}{l}\text { Duration: maximum } 5 \text { days or until patient weaned } \\
\text { from vasopressors } \\
n=388\end{array}$ & $\begin{array}{l}\text { Duration: up to } 281 \text { days } \\
n=60\end{array}$ \\
\hline Primary outcome & 28-day mortality & Hospital mortality \\
\hline
\end{tabular}

1 E.g., Intracranial hypertension, allergic angioedema

2 In this study, indication for vasopressor was not collected and patients who did not have septic shock when admitted to the intensive care unit could have received vasopressors for septic shock after the admission date

$\mathrm{MAP}=$ mean arterial pressure

GRADE evidence regarding recommendation factors, ${ }^{25}$ the Canadian Critical Care Society and the Scandinavian Society of Anaesthesiology and Intensive Care Medicine suggest using lower (MAP 60-70 $\mathrm{mmHg}$ ) rather than higher (MAP 75-85 $\mathrm{mmHg}$ ) blood pressure targets in adult critically ill patients with hypotension requiring vasopressors (conditional recommendation, low confidence in the overall evidence). ${ }^{26,27}$

Blood pressure values measured in the context of clinical trials $^{28}$ or observational studies ${ }^{29}$ are, on average, consistently $>70 \mathrm{mmHg}$. Vasopressor stewardship-i.e., tighter control of vasopressor infusions for all patients, including older patients and those who suffer from chronic hypertension - is the expected impact of this systematic review and related guidelines.

Strengths of this review include explicit and prespecified eligibility criteria, a comprehensive literature search, duplicate adjudication of eligibility, data extraction and risk of bias assessment, prespecified analyses and subgroups, and the use of GRADE to assess and communicate confidence in the effect estimates. Nevertheless, a number of limitations preclude definitive 


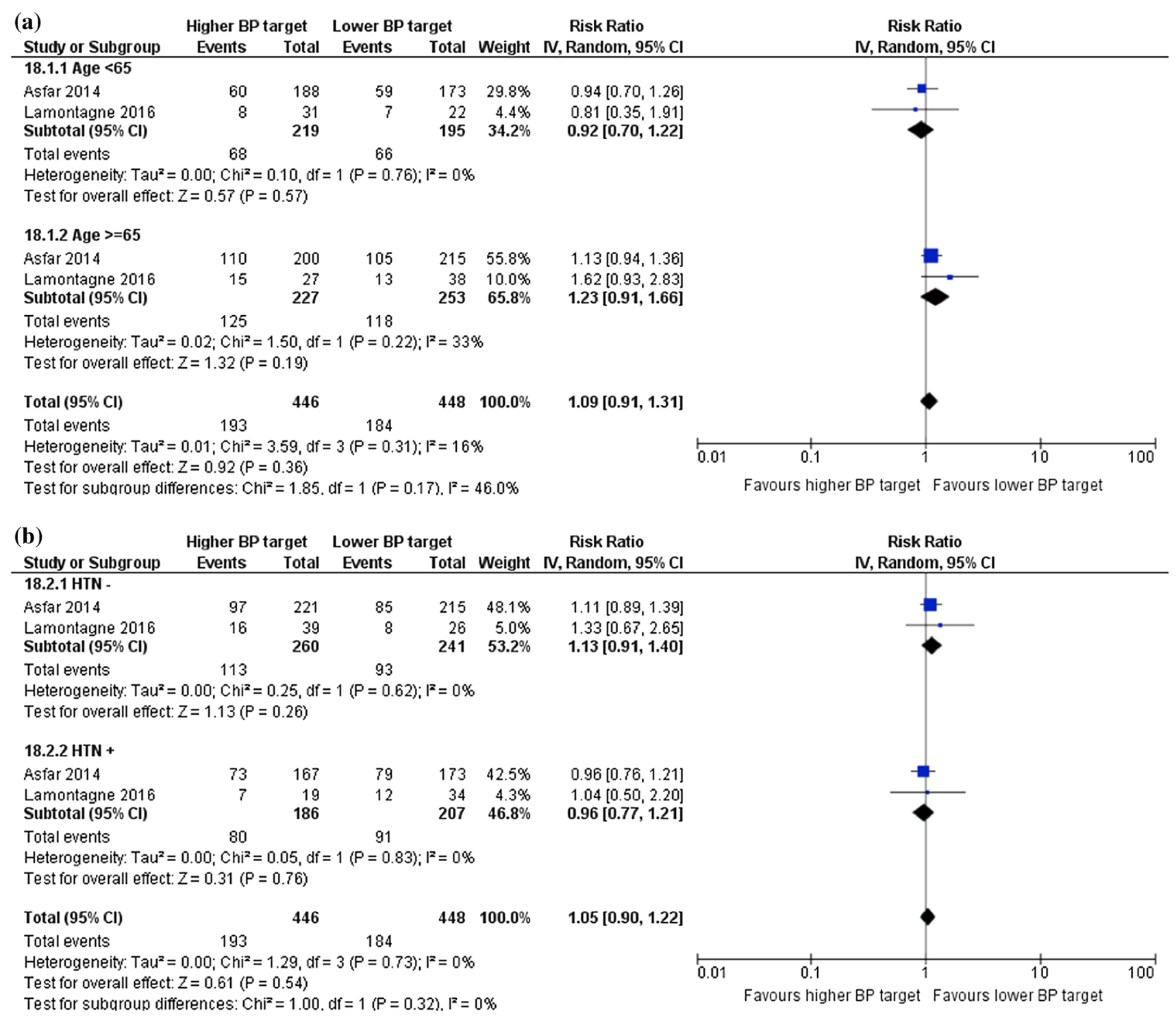

Fig. 2 Forest plots for short-term mortality overall and by subgroups

conclusions regarding the speculative benefits of individualized vasopressor therapy. Confidence intervals and trial sequential analyses confirm that the effect estimates for all outcomes remain imprecise. As such, important benefit and harm remain possible. The scope of the available evidence is limited to the outcomes that were collected and the population targeted by the included trials (i.e., distributive shock). Certain patientimportant outcomes that were not measured in the included studies could influence clinical decisions regarding titration of vasopressors, in particular, postdischarge global quality of life. ${ }^{24}$ In both studies, actual
MAP values were higher than specified in the protocol for the lower target groups. It is conceivable that effect estimates may have been different if vasopressors had been used more sparingly in the lower blood pressure target groups. What is the lowest tolerable blood pressure below which the benefits of vasopressors clearly outweigh the risk of adverse effects? What individual patient characteristics, including the etiology of hypotension, bear on the riskbenefit of vasopressor therapy? Would the effect of higher MAP targets be different with non-catecholamine vasopressors? Adequately powered clinical trials are required to address these persistent knowledge gaps. 
Table 2 GRADE Summary of effects

\begin{tabular}{|c|c|c|c|c|c|}
\hline \multirow{2}{*}{$\begin{array}{l}\text { Outcome } \\
\text { Timeframe }\end{array}$} & \multirow{2}{*}{$\begin{array}{l}\text { Study results and } \\
\text { measurements }\end{array}$} & \multicolumn{2}{|c|}{ Absolute effect estimates } & \multirow{2}{*}{$\begin{array}{l}\text { Confidence in effect } \\
\text { estimates } \\
\text { (Quality of evidence) }\end{array}$} & \multirow[t]{2}{*}{ Summary } \\
\hline & & $\begin{array}{l}\text { Lower MAP } \\
\text { target }(60-70 \\
\text { mmHg) }\end{array}$ & $\begin{array}{l}\text { Higher MAP } \\
\text { target }(75-85 \\
\mathrm{mmHg})\end{array}$ & & \\
\hline $\begin{array}{l}\text { Short-term } \\
\text { Mortality } \\
\text { (90 days) }\end{array}$ & $\begin{array}{l}\text { Relative risk, } 1.05 \\
95 \% \text { CI, } 0.9 \text { to } 1.23 \\
\text { Based on data from }\end{array}$ & $\begin{array}{l}\text { 411/1,000 } \\
\text { Difference: } 2 \\
(95 \% \mathrm{CI}, 41 \mathrm{f}\end{array}$ & $\begin{array}{l}432 / 1,000 \\
\text { nore per } 1,000 \\
\text { er to } 94 \text { more) }\end{array}$ & $\begin{array}{l}\text { Low } \\
\text { Due to imprecision and } \\
\text { risk of bias }\end{array}$ & $\begin{array}{l}\text { Higher MAP target }(75-85 \mathrm{mmHg}) \\
\text { probably has little or no difference } \\
\text { on short-term mortality }\end{array}$ \\
\hline
\end{tabular}

\begin{tabular}{|c|c|}
\hline & $\begin{array}{l}894 \text { patients in } 2 \\
\text { studies }\end{array}$ \\
\hline Long-term & Relative risk, 1.13; \\
\hline Mortality $>90$ & $95 \% \mathrm{CI}, 0.72$ to 1.7 \\
\hline & $\begin{array}{l}\text { Based on data from } \\
118 \text { patients in } 1 \\
\text { study }\end{array}$ \\
\hline Use of Renal & Relative risk, 0.96; \\
\hline Replacement & $95 \%$ CI, 0.80 to 1. \\
\hline Therapy & Based on data from \\
\hline 28 days & $\begin{array}{l}894 \text { patients in } 2 \\
\text { studies }\end{array}$ \\
\hline
\end{tabular}

Ventilator Free Days

(28 days)

Digit, Limb, or Skin Ischemia

Supraventricular

arrhythmia

Relative risk, 0.94;

95\% CI, 0.41 to 2.16

Based on data from 893 patients in 2 studies

Relative risk, 2.08; 95\% CI, 1.28 to 3.38

Based on data from 893 patients in 2 studies

Ventricular
arrhythmia

Relative risk, 1.57;

95\% CI, 0.62 to 4.01

Based on data from 893 patients in 2 studies

Myocardial

Ischemia

Relative risk, 1.45;

95\% CI, 0.37 to 5.71

Based on data from

893 patients in 2 studies
Mesenteric
Relative risk, 0.85;
Based on data from 893 patients in 2 studies
(95\% CI, 18 fewer to 26 more)

367/1,000

414/1,000

Difference: 48 more per 1,000

(95\% CI, 103 fewer to 282 more)

Low

Due to imprecision and risk of bias

Higher MAP target $(75-85 \mathrm{mmHg})$ probably has little or no difference on long-term mortality $>90$ days

\section{7/1,000 343/1,000 Low}

Difference: 14 fewer per $\mathbf{1 , 0 0 0}$

(95\% CI, 71 fewer to 50 more)

Due to imprecision and risk of bias

Higher MAP target (75-85 mmHg) probably has little or no difference on use of renal replacement therapy

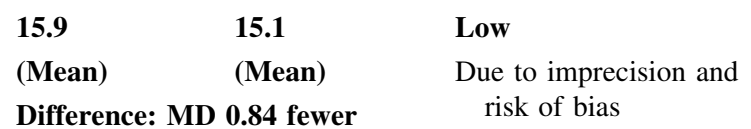

(95\% CI, 2.28 fewer to 0.6 more)

\section{7/1,000 25/1,000 Very Low}

Difference: 2 fewer per $\mathbf{1 , 0 0 0}$

(95\% CI, 16 fewer to 31 more)

47/1,000 98/1,000

Difference: 51 more per 1,000

(95\% CI, 13 more to 112 more)

Due to very serious imprecision and risk of bias

\section{Moderate}

Due to risk of bias

\section{$16 / 1,000 \quad 25 / 1,000 \quad$ Low}

Difference: 9 more per 1,000

(95\% CI, 6 fewer to 47 more)

29/1,000 42/1,000

Difference: 13 more per 1,000

(95\% CI, 18 fewer to 137 more)

Due to serious risk of bias and serious imprecision

\section{Very Low}

Due to very serious imprecision and risk of bias

\section{Very Low}

Due to very serious imprecision and risk of bias
Higher MAP target (75-85 mmHg) probably has little or no difference on ventilator-free days

We are uncertain whether higher MAP target $(75-85 \mathrm{mmHg})$ increases or decreases digit, limb, or skin ischemia

Higher MAP target $(75-85 \mathrm{mmHg})$ increases risk of supraventricular arrhythmia

We are uncertain whether higher MAP target $(75-85 \mathrm{mmHg})$ increases or decreases risk of ventricular arrhythmia

We are uncertain whether higher MAP target $(75-85 \mathrm{mmHg}$ ) increases or decreases myocardial ischemia

We are uncertain whether higher MAP target $(75-85 \mathrm{mmHg})$ increases or decreases mesenteric ischemia

$\mathrm{CI}=$ confidence interval; GRADE = Grading of Recommendations Assessment, Development and Evaluation; MAP = mean arterial pressure; $\mathrm{MD}=$ mean difference 


\section{Conclusion}

Current evidence does not support a MAP target $>70$ $\mathrm{mmHg}$ in critically ill hypotensive patients requiring vasopressor therapy. More randomized-controlled trial data are required to rule on the theoretical benefits of individualized vasopressor dosing.

Acknowledgements The authors are indebted to Jean Maragno and Lois Cottrell for their guidance in designing and carrying out our search strategy.

Conflicts of interest None declared. The authors report no commercial or non-commercial affiliation perceived as a conflict of interest for this work.

Editorial responsibility This submission was handled by Dr. Hilary P. Grocott, Editor-in-Chief, Canadian Journal of Anesthesia.

Author contributions Mathieu Hylands and François Lamontagne contributed substantially to all aspects of this manuscript, including conception and design; acquisition, analysis, and interpretation of data and drafting the article. Morten Hylander Moller, Pierre Asfar, Émilie Belley-Côté, Frédérick D'Aragon, Jon Henrik Laake, Reed Alexander Siemieniuk, Emmanuel Charbonney, François Lauzier, Bram Rochwerg, Per Olav Vandvik, and Gordon Guyatt contributed substantially to the conception and design of the manuscript. Pierre Asfar, Augustin Toma, Anne Julie Frenette, Nicolas Beaudoin, and Joey Kwong contributed substantially to the acquisition of data. Morten Hylander Moller contributed to the analysis of data. Anne Julie Frenette, Émilie Belley-Côté, Frédérick D'Aragon, Jon Henrik Laake, Reed Alexander Siemieniuk, Emmanuel Charbonney, Bram Rochwerg, Per Olav Vandvik, and Gordon Guyatt conntributed substantially to the interpretation of data. Mathieu Hylands and François Lamontagne drafted the manuscript. Morten Hylander Moller, Pierre Asfar, Anne Julie Frenette, Émilie Belley-Côté, Frédérick D'Aragon, Jon Henrik Laake, Reed Alexander Siemieniuk, Emmanuel Charbonney, François Lauzier, Bram Rochwerg, Per Olav Vandvik, and Gordon Guyatt revised and made substantial contributions to the manuscript.

Funding Dr. Lamontagne received a research grant from the Fonds de recherche du Québec - Santé.

\section{Appendix}

Medline Search strategy

1. exp shock/ or shock.mp.

2. (multiple adj3 organ adj3 failure). mp.

3. (shock adj3 cardiogenic). mp.

4. (shock adj3 hemorrhagic). mp.

5. (shock adj3 surgical). mp.

6. (shock adj3 traumatic). mp.

7. (systemic inflammatory adj3 response adj3 syndrome). mp.

8. *Hypotension/ or resuscitation/ or critical care/

9. 1 or 2 or 3 or 4 or 5 or 6 or 7 or 8

10. exp vasoconstrictor agents/ or exp vasopressins/

11. (vasoconstrictive adj3 agents). mp.
12. exp cardiotonic agents/

13. (cardiotonic adj3 agent).mp.

14. (vasopressor or vasoconstrictor). $\mathrm{mp}$.

15. exp catecholamines/ or catecholamine.mp.

16. exp epinephrine/ or epinephrine.mp.

17. adrenaline.mp.

18. norepinephrine/ or norepinephrine.mp.

19. noradrenaline.mp.

20. exp isoproterenol/ or isoprenaline.mp.

21. exp metaproterenol/ or orciprenaline.mp.

22. exp ephedrine/ or ephedrine.mp.

23. exp phenylephrine/ or phenylephrine.mp.

24. exp dopamine agents/ or exp dopamine/ or exp dopamine agonists or dopamine.mp.

25. dobutamine.mp. or exp dobutamine/

26. vasopressin.mp. or exp vasopressins/

27. arginine vasopressin.mp or exp arginine vasopressin/

28. desmopressin.mp. or exp deamino arginine vasopressin/

29. lyoressub.mp. exp lypressin/

30. ornipressin.mp. or exp ornipressin/

31. terlipressin.mp.

32. (glypressin or pitressin). mp.

33. felypressin.mp. or exp felypressin/

34. synephrine.mp. or exp synephrine/

35. or/10-34

36. 9 and 35

37. ret*.mp.

38. randomized controlled trial.mp. or exp Randomized Controlled Trial/

39. (randomized adj3 control : adj3 trial :).mp.

40. exp Controlled Clinical Trial/

41. (controlled adj3 clinical adj3 trial).mp.

42. (clinical adj3 trial :).mp.

43. (experimental adj3 trial :).mp.

44. exp Clinical Study/

45. (clinical adj3 study).mp.

46. Comparative Study/

47. (comparative adj3 study). mp.

48. exp Evaluation Studies/

49. (evaluation adj3 study).mp.

50. exp Multicenter Study/

51. (multicenter adj3 study).mp.

52. exp Observational Study/ or prospective studies/ or retrospective studies/

53. (observational adj3 study).mp.

54. exp Case-Control Studies/

55. (case adj3 control adj3 studies).mp.

56. exp Cohort Studies/

57. (cohort adj3 studies).mp.

58. or $/ 37-57$

59. 36 and 58

60. (animals not (humans and animals)).sh. 59 and 60 
Clinical experiments excluded from this review

\begin{tabular}{ll}
\hline Author, year & Title \\
\hline Larsson, ongoing & The Effects of Blood Pressure on Renal Function and \\
ClinicalTrials.gov: & Oxygenation in Septic Shock
\end{tabular}

NCT02453425

Pettilä, ongoing ClinicalTrials.gov:

NCT02579525

Targeted Tissue Perfusion Versus Macrocirculatory-guided Standard Care in Patients With Septic Shock (TARTARE2S)

Bourgouin, 2005

Increasing mean arterial pressure in patients with septic shock: effects on oxygen variables and renal function

Deruddre, 2007

Suk, 2007

Renal arterial resistance in septic shock: effects of increasing mean arterial pressure with norepinephrine on the renal

\section{Description/Reason for Exclusion}

Crossover trial comparing norepinephrine titrated to a MAP of 60,75 , or $90 \mathrm{mmHg}$. By design, each blood pressure target is achieved for a duration of $30 \mathrm{~min}$. The primary outcome measure is glomerular filtration rate

\section{Reason for exclusion:}

Wrong timing (duration of intervention is $<24 \mathrm{hr}$ by design)

RCT comparing different treatment protocols to guide care in patients with septic shock

In the intervention group, care is guided by "targeted tissue perfusion": capillary refill time, peripheral temperature, mottling, and diuresis, with a MAP inferior safety limit of $50-65 \mathrm{mmHg}$ (or $65-70 \mathrm{mmHg}$ in chronically hypertensive patients)

In the control group, care is guided by "macocirculatory targets": CVP, diuresis, $\mathrm{SvO}_{2}$, and a blood pressure target of $65-75 \mathrm{mmHg}$ (or $75-80 \mathrm{mmHg}$ in chronically hypertensive patients)

\section{Reason for exclusion:}

Wrong intervention/comparator. Although the blood pressure specified in each group is different, the experimental group is exposed to a number of interventions wherein the lower blood pressure consists of a safety threshold rather than a target. Indeed, interventions may be administered to achieve higher blood pressures in the experimental group according to other "targeted tissue perfusion" markers

RCT comparing titration of norepinephrine with a target MAP of 65 or $85 \mathrm{mmHg}$ in septic shock patients. By design, the experimental blood pressure target was maintained for a duration of $4 \mathrm{hr}$. Outcome measures consisted of hemodynamic and metabolic variables

\section{Reason for exclusion:}

Wrong timing (duration of intervention $<24 \mathrm{hr}$ by design) resistive index assessed with Doppler ultrasonography

Prospective study comparing MAP targets of 65,75 , and 85 $\mathrm{mmHg}$ in septic shock patients. Each target threshold was maintained for consecutive, nonrandomized periods of $2 \mathrm{hr}$ in duration. Outcome measures were hemodynamic parameters, renal function and renal resistive index assessed by Doppler

\section{Reason for exclusion:}

Wrong timing (duration of intervention $<24 \mathrm{hr}$ by design)

RCT comparing MAP targets of $65 \mathrm{mmHg}$ or $85 \mathrm{mmHg}$ in septic shock patients. By design, the target blood pressure threshold was maintained for a 6-hr duration. Outcome measures were lactate metabolism and hemodynamic variables

Reason for exclusion:

Wrong timing (duration of intervention $<24 \mathrm{hr}$ by design)

$\mathrm{RCT}=$ randomized-controlled trial; $\mathrm{MAP}=$ mean arterial pressure; $\mathrm{CVP}=$ central venous pressure; $\mathrm{SvO}_{2}=$ central venous oxygen saturation 
Risk of bias assessment

\begin{tabular}{lll}
\hline & Asfar 2014 & Lamontagne 2016 \\
\hline Random sequence generation & Low & Low \\
Allocation concealment & Low & Low \\
Blinding & High & High \\
Incomplete outcome data (primary outcome) & Low & Low \\
Incomplete outcome data (other outcomes) & Low & Unclear (likely low) \\
Selective outcome reporting (primary outcome) & Low & Low \\
Selective outcome reporting (other outcomes) & Low & Low \\
Other risks of bias & Low & Low \\
\hline
\end{tabular}

1 Secondary outcomes were not screened systematically

Trial Sequential Analyses (TSA) (risk of random errors)

90-day mortality, 2 trials $(n=894)$

$20 \%$ relative risk difference (RRD), control event proportion $41 \%$, alpha $5 \%$, beta $20 \%$

TSA adjusted RR, $1.05 ; 95 \% \mathrm{CI}, 0.88$ to 1.26

TSA shows that $82 \%(894 / 1,088)$ of the required information size needed to detect or reject a $20 \%$ RRD in mortality has been accrued. The cumulative $\mathrm{Z}$ curve does not touch the conventional boundary for benefit or harm $(P<0.05)$ or the trial sequential monitoring boundary for benefit or harm, and the boundary for futility has not been crossed.

Interpretation: no statistically significant difference (imprecision).

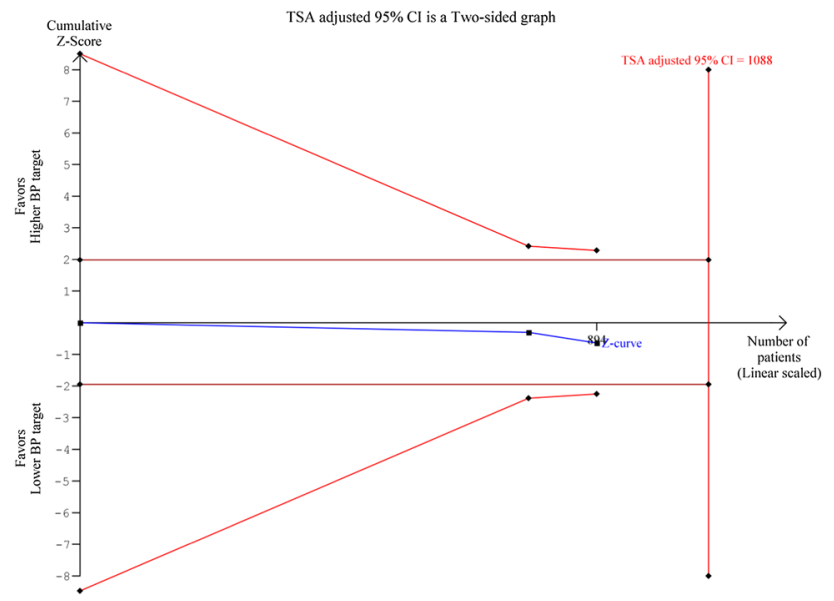

Use of renal replacement therapy (RRT), 2 trials $(n=894)$

$20 \%$ RRD, control event proportion $35 \%$, alpha $5 \%$, beta $20 \%$

TSA adjusted RR, 0.93 ; $95 \%$ CI, 0.62 to 1.40

TSA shows that $39 \%(894 / 2,278)$ of the required information size needed to detect or reject a $20 \%$ RRD in use of RRT has been accrued. The cumulative $\mathrm{Z}$ curve does not touch the conventional boundary for benefit or harm $(P<0.05)$ or the trial sequential monitoring boundary for benefit or harm, and the boundary for futility has not been crossed.

Interpretation: no statistically significant difference (imprecision).

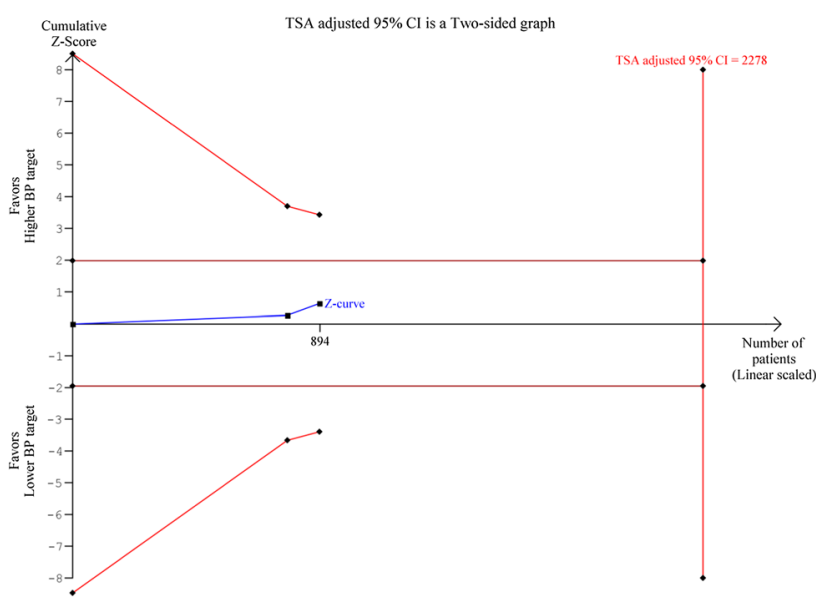

Duration of renal replacement therapy, two trials $(n=894)$

$20 \%$ RRD, alpha $5 \%$, beta $20 \%$

TSA adjusted RR, $-0.41 ; 95 \% \mathrm{CI},-3.91$ to 3.09

TSA shows that $15 \%(894 / 6,161)$ of the required information size needed to detect or reject a $20 \%$ RRD in duration of RRT has been accrued. The cumulative $\mathrm{Z}$ curve 
does not touch the conventional boundary for benefit or harm $(P<0.05)$ or the trial sequential monitoring boundary for benefit or harm, and the boundary for futility has not been crossed.

Interpretation: no statistically significant difference (severe imprecision).

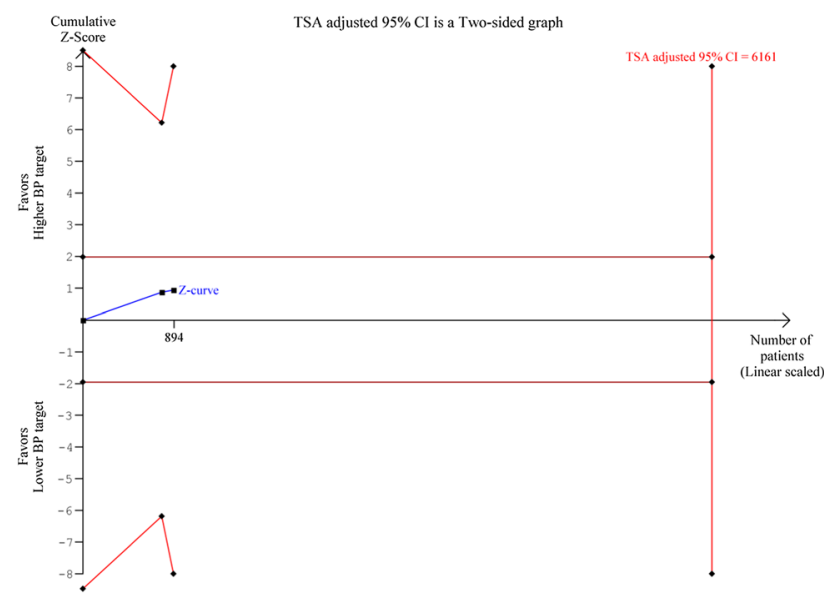

Duration of mechanical ventilation (MV), two trials $(n=894)$

$20 \%$ RRD, alpha $5 \%$, beta $20 \%$

TSA adjusted RR, $0.00 ; 95 \% \mathrm{CI},-0.84$ to 0.84

TSA shows that $77 \%(894 / 1,162)$ of the required information size needed to detect or reject a $20 \%$ RRD in duration of MV has been accrued. The cumulative $\mathrm{Z}$ curve does not touch the conventional boundary for benefit or harm $(P<0.05)$ or the trial sequential monitoring boundary for benefit or harm, and the boundary for futility has not been crossed.

Interpretation: no statistically significant difference (imprecision).

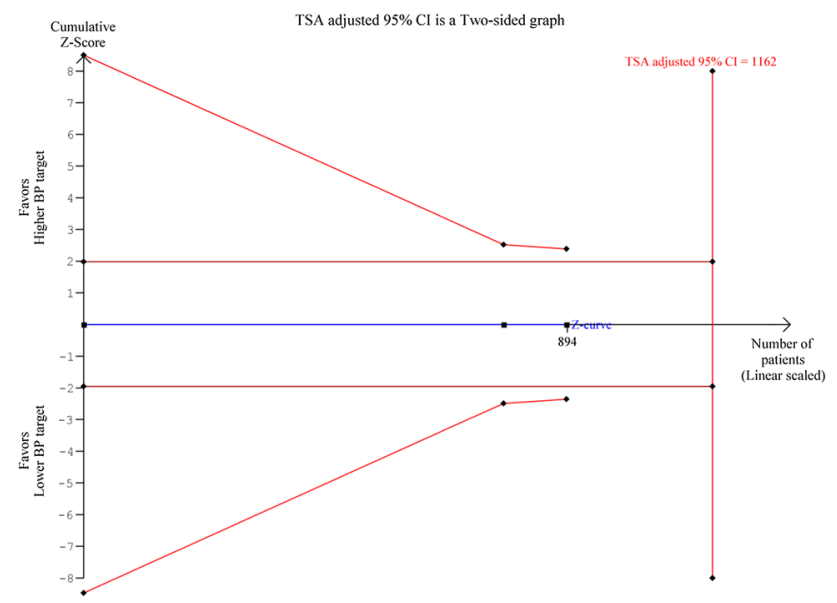

Supraventricular arrhythmias, 2 trials $(n=894)$

$20 \%$ RRD, control event proportion $5 \%$, alpha $5 \%$, beta $20 \%$

TSA adjusted RR, 2.08; $95 \%$ CI, 1.29 to 3.41

TSA shows that $7 \%(894 / 13,493)$ of the required information size needed to detect or reject a $20 \%$ RRD in supraventricular arrhythmia has been accrued. The cumulative $\mathrm{Z}$ curve crosses the conventional boundary for benefit or harm $(P<0.05)$ and the trial sequential monitoring boundary for benefit or harm.

Interpretation: a statistically significant difference (severe imprecision).

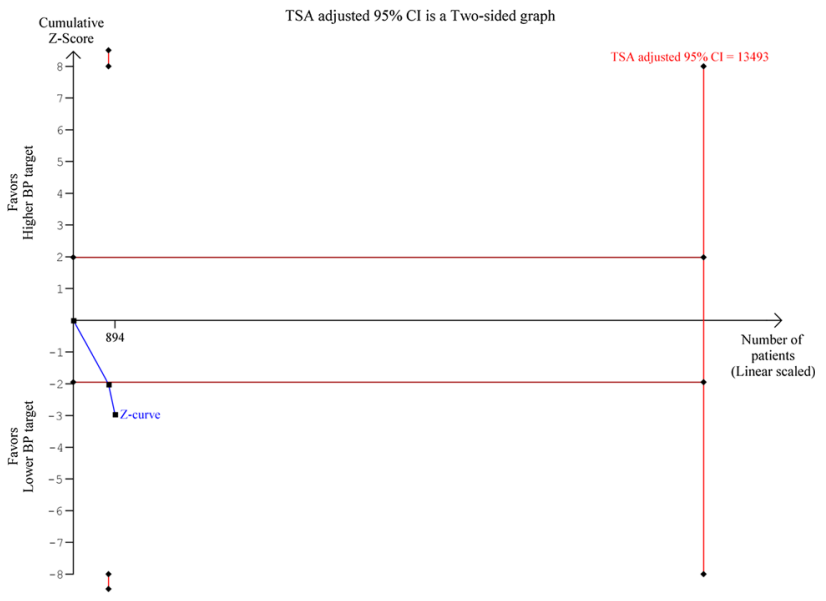

Ventricular arrhythmia, 2 trials $(n=894)$

$20 \%$ RRD, control event proportion $2 \%$, alpha $5 \%$, beta $20 \%$

TSA adjusted RR, 1.57; 95\% CI, 0.62 to 4.04

Less than $5 \%(894 / 34,685)$ of the required information size needed to detect or reject a $20 \%$ RRD in ventricular arrhythmia has been accrued. Consequently, no TSA plot can be prepared.

Interpretation: severe imprecision

Digit, limb, or skin ischemia, 2 trials $(n=894)$

$20 \%$ RRD, control event proportion $3 \%$, alpha $5 \%$, beta $20 \%$

Less than $5 \%(894 / 22,911)$ of the required information size needed to detect or reject a $20 \%$ RRD in digit, limb, or skin ischemia has been accrued. Consequently, no TSA plot or TSA-adjusted 95\% CI can be prepared.

Interpretation: severe imprecision 
Mesenteric ischemia, 2 trials $(n=894)$

$20 \%$ RRD, control event proportion $3 \%$, alpha $5 \%$, beta $20 \%$

Less than $5 \%(894 / 22,911)$ of the required information size needed to detect or reject a $20 \%$ RRD in mesenteric ischemia has been accrued. Consequently, no TSA plot or TSA-adjusted $95 \%$ CI can be prepared.

Interpretation: severe imprecision

Mesenteric ischemia, 2 trials $(n=894)$

$20 \%$ RRD, control event proportion $3 \%$, alpha $5 \%$, beta $20 \%$

Less than $5 \%(894 / 82,043)$ of the required information size needed to detect or reject a $20 \%$ RRD in myocardial ischemia has been accrued. Consequently, no TSA plot or TSA-adjusted 95\% CI can be prepared.

Interpretation: severe imprecision

\section{References}

1. Adhikari NK, Fowler RA, Bhagwanjee S, Rubenfeld GD. Critical care and the global burden of critical illness in adults. Lancet 2010; 376: 1339-46.

2. Lamontagne $F$, Cohen D, Herridge $M$. Understanding patientcentredness: contrasting expert versus patient perspectives on vasopressor therapy for shock. Intensive Care Med 2016. DOI:10. 1007/s00134-016-4518-x.

3. Andreis DT, Singer M. Catecholamines for inflammatory shock: a Jekyll-and-Hyde conundrum. Intensive Care Med 2016; 42: 1387-97.

4. Dellinger RP, Levy MM, Rhodes A, et al. Surviving sepsis campaign: international guidelines for management of severe sepsis and septic shock: 2012. Crit Care Med 2013; 41: 580-637.

5. Schmittinger CA, Torgersen C, Luckner G, Schroder DC, Lorenz $I$, Dunser $M W$. Adverse cardiac events during catecholamine vasopressor therapy: a prospective observational study. Intensive Care Med 2012; 38: 950-8.

6. D'Aragon F, Belley-Cote EP, Meade MO, et al. Blood pressure targets for vasopressor therapy: a systematic review. Shock 2015; 43: 530-9.

7. Institute of Medicine; Committee on Standards for Developing Trustworthy Clinical Practice Guidelines. Clinical practice guidelines we can trust. Washington, DC: National Academies Press; 2011.

8. Siemieniuk RA, Agoritsas T, Macdonald H, Guyatt GH, Brandt L, Vandvik $P O$. Introduction to BMJ rapid recommendations. BMJ 2016; 354: i5191.

9. Moher D, Liberati A, Tetzlaff J. Altman DG; PRISMA Group. Preferred reporting items for systematic reviews and metaanalyses: the PRISMA statement. J Clin Epidemiol 2009; 62: 1006-12.

10. DerSimonian $R$, Laird $N$. Meta-analysis in clinical trials. Control Clin Trials 1986; 7: 177-88.

11. Deeks JJ. Issues in the selection of a summary statistic for metaanalysis of clinical trials with binary outcomes. Stat Med 2002; 21: $1575-600$.
12. ARISE Investigators; ANZICS Clinical Trials Group; Peake SL, Delaney A, Bailey $M$, et al. Goal-directed resuscitation for patients with early septic shock. The New England journal of medicine 2014; 371: 1496-506.

13. Bassler D, Briel M, Montori VM, et al. Stopping randomized trials early for benefit and estimation of treatment effects: systematic review and meta-regression analysis. JAMA 2010; 303: 1180-7.

14. Guyatt G, Busse J. Modification of Cochrane Tool to assess risk of bias in randomized trials. 2016. Available from URL: http:// distillercer.com/resources/ (accessed March 2017).

15. Guyatt GH, Oxman AD, Vist GE, et al. GRADE: an emerging consensus on rating quality of evidence and strength of recommendations. BMJ 2008; 336: 924-6.

16. Cochrane Handbook. Recommendations on testing for funnel plot asymmetry. Available from URL: http://handbook.cochrane. org/chapter_10/10_4_3_1_recommendations_on_testing_for_ funnel_plot_asymmetry.htm (accessed March 2017).

17. Guyatt GH, Oxman AD, Vist $G$, et al. GRADE guidelines: 4. Rating the quality of evidence-study limitations (risk of bias). J Clin Epidemiol 2011; 64: 407-15.

18. Guyatt GH, Oxman AD, Kunz R, et al. GRADE guidelines 6 . Rating the quality of evidence-imprecision. J Clin Epidemiol 2011; 64: 1283-93.

19. Guyatt GH, Oxman AD, Kunz R, et al. GRADE guidelines: 7 . Rating the quality of evidence-inconsistency. J Clin Epidemiol 2011; 64: 1294-302.

20. Guyatt GH, Oxman AD, Kunz, R, et al. GRADE guidelines: 8. Rating the quality of evidence-indirectness. J Clin Epidemiol 2011; 64: 1303-10.

21. Guyatt $G H$, Oxman AD, Montori $V$, et al. GRADE guidelines: 5 . Rating the quality of evidence-publication bias. J Clin Epidemiol 2011; 64: 1277-82.

22. Brok J, Thorlund $K$, Gluud $C$, Wetterslev J. Trial sequential analysis reveals insufficient information size and potentially false positive results in many meta-analyses. J Clin Epidemiol 2008; 61: 763-9.

23. Asfar P, Meziani F, Hamel JF, et al. High versus low bloodpressure target in patients with septic shock. N Engl J Med 2014; 370: 1583-93.

24. Lamontagne $F$, Meade $M O$, Hebert PC, et al. Higher versus lower blood pressure targets for vasopressor therapy in shock: a multicentre pilot randomized controlled trial. Intensive Care Med 2016; 42: 542-50.

25. Alonso-Coello P, Oxman AD, Moberg J, et al. GRADE Evidence to Decision (EtD) frameworks: a systematic and transparent approach to making well informed healthcare choices. 2: Clinical practice guidelines. BMJ 2016; 353: i2089.

26. Rochwerg B, Hylands $M$, Moller $M$, et al. CCCS-SSAI WikiRecs Clinical Practice Guideline: vasopressor blood pressure targets in critically ill adults with hypotension. Can J Anesth 2017; 64: this issue. DOI: $10.1007 / \mathrm{s} 12630-017-0878-0$

27. Rochwerg B, Hylands M, Moller M, et al. CCCS-SSAI WikiRecs Clinical Practice Guideline: vasopressors in early traumatic shock. Can J Anesth 2017; 64: this issue. DOI: 10.1007/ s12630-017-0879-z

28. Russell JA, Walley KR, Singer J, et al. Vasopressin versus norepinephrine infusion in patients with septic shock. N Engl $\mathrm{J}$ Med 2008; 358: 877-87.

29. Lamontagne F, Cook DJ, Meade MO, et al. Vasopressor use for severe hypotension-a multicentre prospective observational study. PloS One 2017; 12: e0167840. 